\title{
False negative antigen tests in dogs infected with heartworm and placed on macrocyclic lactone preventives
}

\author{
Jason Drake ${ }^{1}$, Jeff Gruntmeir ${ }^{2}$, Hannah Merritt ${ }^{3}$, Lynn Allen ${ }^{1}$ and Susan E Little 2* $^{*}$
}

\begin{abstract}
Background: Dogs with chronic inflammation, including those with heartworm being managed with macrocyclic lactones and doxycycline (slow kill, SK), may develop immune complexes that block detection of Dirofilaria immitis antigen on commercial tests.

Methods: To determine if SK could result in development of false-negative antigen tests, we collected serum samples from dogs that had been diagnosed with heartworm by antigen detection, with or without confirmation by detection of D. immitis microfilariae, placed on monthly macrocyclic lactones and doxycycline, and that later tested negative on an antigen test, and then tested them for antigen of $D$. immitis before and after treatment to disrupt immune complexes.

Results: Serum samples from a total of 15 dogs managed with SK were negative for antigen prior to heating on commercial assay (DiroCHEK ${ }^{\oplus}$, Zoetis) by colorimetric detection and spectrophotometry, but after heat treatment, 8/15 (53.3\%) samples converted to positive. Review of the medical records of each dog indicated that, after the heartworm diagnosis, only 7/15 (46.7\%) dogs appeared to receive preventive monthly as prescribed, including 3 dogs that had detectable antigen after heating the sample and 4 dogs that did not have detectable antigen after heating. Whole blood was available from 9 dogs; microfilariae of $D$. immitis were detected in 1 sample.

Conclusions: These data suggest that immune complex formation in dogs infected with heartworm and managed with SK can induce false negative antigen test results, misleading veterinarians and owners about the efficacy of this approach. Moreover, compliance with preventive administration appears poor, even after a heartworm diagnosis. The presence of persistent microfilaremia in at least one dog has implications for resistance selection.
\end{abstract}

Keywords: Dirofilaria immitis, False-negative antigen test, Immune complexes, Slow kill

\section{Background}

Slow kill (SK) is defined as the use of monthly macrocyclic lactone heartworm preventives, with or without doxycycline, in lieu of melarsomine dihydrochloride (Immiticide ${ }^{\circ}$, Merial) in dogs infected with adult Dirofilaria immitis [1]. Historically, veterinarians have used SK to manage adult heartworm infections for a variety of reasons, including when the approved adulticide is not available, when the cost of adulticide treatment is rejected by a dog owner, or when the medical risk of

\footnotetext{
* Correspondence: susan.little@okstate.edu

${ }^{2}$ Department of Veterinary Pathobiology, Center for Veterinary Health Sciences, Oklahoma State University, Room 25074078 McElroy Hall, Stillwater, OK, USA

Full list of author information is available at the end of the article
}

adulticide therapy is thought to be too great. The practice of SK is explicitly discouraged within the guidelines of both the American Heartworm Society (AHS) and the Companion Animal Parasite Council (CAPC) [1,2]. The European Scientific Counsel Companion Animal Parasites (ESCCAP) also explicitly does not recommend the use of SK-like protocols [3].

Several reasons to avoid SK have been described, including compliance failure and selection for resistance [4]. The adult worms, which may be $>30 \mathrm{~cm}$ in length, reside in the pulmonary arteries and cause severe inflammation, truncation of pulmonary vessels, thickening of the arterial parenchyma, pulmonary hypertension and 
right heart failure [5-9]. Utilization of the approved adulticide, melarsomine dihydrochloride, is an effective method for elimination of adult heartworms [10], while SK methods of continuous monthly use of preventive doses of macrocyclic lactones, with or without the addition of doxycycline, result in worm death and elimination over a lengthy and somewhat unpredictable period of time $[11,12]$. Efficacy of SK is poor (56.3\%) against 8-month-old worms when monthly low-dose ivermectin is administered for 16 consecutive months [11] but improves (94.9\%) when administered consistently for 29 consecutive months [12]. However, SK allows worms to continue to reside within the pulmonary arteries, prolonging the inflammatory process and allowing further disease progression [4]. Indeed, heartworm-infected dogs administered SK for a one-year period developed radiographic signs of heartworm disease and severe pulmonary pathology with multiple pathologic changes including enlarged pulmonary arteries, villous proliferation characteristic of heartworm infection, alveolar disease, interstitial lung disease, and parenchymal fibrosis [4].

In clinical practice and in clinical research projects, veterinarians may use antigen testing as a proxy for SK efficacy $[13,14]$. The relationship between antigen status, microfilaremia, and the presence of adult worms in dogs managed with SK has not been well defined. Interestingly, in the original publication describing the prolonged death of adult heartworms during monthly administration of preventive, antigen levels decreased during administration of ivermectin to heartworm-infected dogs even though worms survived, suggesting the process of SK interferes with either antigen production by $D$. immitis or antigen detection by commercial tests [11].

All currently available heartworm preventives contain macrocyclic lactones as the active ingredient against D. immitis [15]. Recently, D. immitis resistant to macrocyclic lactone preventives have been described in North America [16-18]. Repeated administration of preventives to dogs infected with adult $D$. immitis may increase the risk of resistance to macrocyclic lactones by increasing selective pressure, placing all dogs, including uninfected dogs maintained on preventives, at higher risk of infection [15]. Although experimental data document that SK methods require more than 1-2 years to eliminate adult heartworms [11,12], some veterinarians have reported dogs converting from $D$. immitis antigen positive to antigen negative status within a few months of starting SK (S. Little, pers. comm.). Recent publications document false negative antigen tests occur in both dogs and cats, presumably due to formation of immune complexes, a phenomenon described in the literature through the 1980s $[19,20]$. Here we report initial data indicating false negative antigen tests are common in dogs on SK.

\section{Methods}

\section{Source of samples}

Serum and EDTA-anticoagulated whole blood samples were requested from canine patients seen by general practitioners that had been previously diagnosed by the participating veterinary practice as infected with $D$. immitis by a commercial in-clinic antigen test, with or without confirmation by microfilaria testing, within the previous 36 months. Specifically, samples were requested from dogs that had not been treated with the label-approved adulticide (melarsomine dihydrochloride), but instead were placed on a monthly macrocyclic lactone-based preventive and doxycycline (SK). A history was collected for each dog and included patient identification information, previous heartworm testing method(s), test timing, test result(s), SK protocol employed, heartworm preventive used, and compliance with heartworm prevention. Compliance with heartworm prevention was evaluated based on date of purchase of heartworm preventive from the clinic. The research protocol was approved by the Novartis Animal Welfare Officer prior to instituting the research and conformed to all requirements and ethical guidelines of both Novartis Animal Health and Oklahoma State University's Center for Veterinary Health Sciences.

\section{Inclusion and exclusion criteria}

Samples included in this study were collected from dogs with a diagnosis of heartworm infection in the past 36 months based on commercial antigen test and that had converted to antigen-negative status within the past 24 months using any commercially approved in-clinic antigen test conducted following the manufacturer's instructions. Serum samples were tested upon receipt to confirm antigen-negative status (see below); only samples confirmed negative upon receipt were included in the study. Samples from dogs treated with melarsomine dihydrochloride and those that could not be confirmed through the medical records to meet the inclusion requirements were excluded from the study. Serum samples $(3-7 \mathrm{~mL})$ were required from each dog enrolled in the study. When available, EDTA-anticoagulated whole blood was also evaluated for microfilariae (see below) but submission of a paired whole blood sample was not required for inclusion.

\section{Antigen testing}

Upon receipt of each serum sample, antigen negative status was confirmed on a microtiter well based assay (DiroCHEK ${ }^{\ominus}$, Zoetis) according to manufacturer's instructions. Only samples that tested negative upon initial evaluation were included in the study. Following confirmation testing, each sample was heat treated and retested as previously described [21]. Briefly, $1 \mathrm{~mL}$ of serum was placed in a $1.5 \mathrm{~mL}$ snap-cap microcentrifuge 
tube, heated to $104^{\circ} \mathrm{C}$ for 10 minutes in a standard laboratory dry heat block, and the resulting coagulum centrifuged at $16,000 \times \mathrm{g}$ for 5 minutes. After centrifugation, the supernatant was removed from the coagulum and tested in triplicate using a microtiter well based assay (DiroCHEK ${ }^{\bullet}$, Zoetis) according to manufacturer's instructions. In addition to colorimetric change, spectrophotometry $(650 \mathrm{~nm})$ was used to record the optical density (O.D.) of each individual sample, before and after heat treatment, on the same microtiter plate using the same positive and negative controls and identical calculated cut-off values [21].

\section{Microfilaria testing}

Modified Knott tests were performed on all dogs for which whole blood samples were available as previously described [22]. Briefly, $9 \mathrm{~mL}$ of $2 \%$ formalin was added to $1 \mathrm{~mL}$ of whole blood, the cells allowed to lyse, the mixture centrifuged at $1,200 \times \mathrm{g}$, and the pellet stained with methylene blue and examined by microscopy for microfilariae. Identify of microfilariae were confirmed by morphology and by PCR and sequencing as previously described [22,23].

\section{Results}

A total of 15 dogs met the enrollment inclusion and exclusion criteria. Serum samples were submitted from all
15 dogs. Antigen testing of the samples post-heating revealed serum from 8 of 15 dogs (53.3\%) that had become antigen negative following SK therapy were actually still antigen positive; samples from 7 of 15 (46.7\%) dogs remained antigen negative after heating (Table 1). Paired anticoagulated whole blood samples were submitted from 9 of 15 dogs; of these, 1/9 (11.1\%) had evidence of microfilariae. Microfilariae were confirmed as D. immitis via morphology and PCR followed by sequencing (data not shown).

Evaluation of medical records from each dog revealed SK was performed using monthly low-dose ivermectin (HeartGard Plus ${ }^{\oplus}$, Merial or IverHeart Plus ${ }^{\circ}$, Virbac) in 10 dogs and monthly topical moxidectin/imidicloprid (AdvantageMulti for Dogs ${ }^{\circ}$, Bayer Animal Health) in 5 dogs (Table 1). Two of the 10 dogs initially prescribed low-dose ivermectin were switched to a monthly preventive containing milbemycin oxime (Trifexis ${ }^{\circ}$, Elanco or Sentinel ${ }^{\circ}$ Flavor Tabs, Novartis Animal Health) following conversion to negative heartworm antigen status. All 15 dogs were prescribed doxycycline at some point for various periods of time; one dog received 6 months of doxycycline, while all other dogs were prescribed single or multiple, intermittent 30d courses of therapy (Table 1). Based on purchase history, 7 of the 15 dogs had evidence in the medical records supporting compliance with monthly preventive as prescribed.

Table 1 Prescribed preventive, administration route, compliance, doxycycline use, and results of modified Knott test and antigen tests on samples collected from 15 heartworm infected dogs managed with slow kill

\begin{tabular}{|c|c|c|c|c|c|c|c|}
\hline Dog ID & $\begin{array}{l}\text { Preventive } \\
\text { prescribed }\end{array}$ & $\begin{array}{l}\text { Route of } \\
\text { administration }\end{array}$ & $\begin{array}{l}\text { Compliant with } \\
\text { dispensing }\end{array}$ & $\begin{array}{l}\text { Doxycycline (10 mg/kg PO) } \\
\text { protocol }\end{array}$ & $\begin{array}{l}\text { Modified } \\
\text { Knott }\end{array}$ & $\begin{array}{l}\text { Pre-heating antigen } \\
\text { test results }\end{array}$ & $\begin{array}{l}\text { Post-heating antigen } \\
\text { test results }\end{array}$ \\
\hline 1 & Moxidectin & Topical & No & $30 d$ & ND & - & + \\
\hline 2 & Moxidectin & Topical & Yes & $30 \mathrm{~d}$, four times & ND & - & + \\
\hline 3 & Moxidectin & Topical & No & $30 \mathrm{~d}$, four times & ND & - & + \\
\hline 4 & Ivermectin & Oral & Yes & 30d, twice & Neg & - & + \\
\hline 5 & Ivermectin & Oral & No & $30 d$ & Neg & - & + \\
\hline 6 & Ivermectin & Oral & No & 30d, twice & Neg & - & + \\
\hline 7 & Ivermectin & Oral & Yes & 30d, twice & Neg & - & + \\
\hline 8 & Ivermectin & Oral & No & $30 d$ & POS* $^{*}$ & - & + \\
\hline 9 & Moxidectin & Topical & Yes & $6 \mathrm{mo}$ & ND & - & - \\
\hline 10 & Moxidectin & Topical & No & 30d, twice & Neg & - & - \\
\hline 11 & Ivermectin & Oral & Yes & $30 d$ & Neg & - & - \\
\hline 12 & Ivermectin & Oral & Yes & 30d, twice & Neg & - & - \\
\hline 13 & Ivermectin & Oral & No & $30 d$ & ND & - & - \\
\hline \multirow[t]{2}{*}{14} & Ivermectin, & Oral & Yes & 30d, twice & ND & - & - \\
\hline & Milbemycin & Oral & & & & & \\
\hline \multirow[t]{2}{*}{15} & Ivermectin, & Oral & No & $30 d$, three times & Neg & - & - \\
\hline & Milbemycin & Oral & & & & & \\
\hline
\end{tabular}




\section{Discussion}

Together, these data indicate that antigen tests may not be a reliable indicator of heartworm antigen status in D. immitis-infected dogs managed with SK; over half the dogs which converted to antigen negative were actually still antigen positive following treatment of serum to disrupt immune complexes. Experimental infection studies have shown that SK prolongs the death of adult worms over 16 months to more than 3 years depending on the age of the worms when the protocol is first initiated $[11,12]$. During SK, inflammation is induced which is radiographically evident and can exacerbate clinical disease $[4,24]$. We suspect that the hypergammaglobulinemic state induced by the reaction to dead and dying worms allows formation of antigen-antibody complexes in some canine patients, masking antigen of $D$. immitis and preventing detection on subsequent assays. A simple heat treatment step prior to antigen assay of serum would be expected to destroy any complexes, if present, and allow detection of antigen, the reason this step was routinely included when heartworm antigen tests were first developed [25,26].

A limitation of the present study is that the dogs studied were pet dogs and therefore no necropsy data or worm counts were available. Although over half the dogs testing negative were actually shown to harbor antigen, we may have been detecting residual antigen in some dogs without the concomitant presence of live, adult worms. Similarly, because a second, microtiter well based confirmatory antigen test was not documented for all dogs prior to starting SK, some dogs which apparently converted to true negative may not have been originally infected. Nonetheless, the results of the present study draw attention to the possibility that false negative antigen test results may commonly develop in dogs on SK, a problem which could mislead veterinarians in clinical practice about the infection status of their patients.

One of the dogs in the present study managed with a SK protocol and initially antigen negative had circulating microfilariae (Table 1), while microfilariae were not detected in the other 8 dogs for which whole blood was available; the microfilaria status of the 6 dogs for which only serum was provided is unknown. These data support previous findings suggesting microfilariae continue to be present in some dogs despite monthly administration of macrocyclic lactones [15]. Although not reproduced experimentally, one potentially catastrophic effect of the use of SK is increased selection for resistance to the macrocyclic lactones, the anthelmintic class of all currently available heartworm preventives [15]. This phenomenon was recently investigated by administering repeated doses of ivermectin to a dog infected with macrocyclic lactone-resistant Dirofilaria immitis, resulting in an apparent shift in the proportion of microfilariae with resistant markers, a result interpreted to be consistent with the effect of increased selective pressure [27]. Some dispute the importance of the resistance concern by citing research showing that third-stage larvae produced from microfilariae harvested from dogs treated with doxycycline are unable to develop to adult worms [28,29]. While important, those experiments have involved a small number of dogs $(\mathrm{n}=1$ or 2$)$ challenged with a small number of larvae $(n=5-50)$. Extending the findings to a large population of infected dogs in the field may be imprudent, particularly given the growing awareness of resistance to macrocyclic lactones in D. immitis in North America.

Interestingly, the present study also documented that compliance with SK was poor despite a heartworm diagnosis and owner awareness of an active heartworm infection. These data confirm what many veterinarians have long suspected: successfully implementing SK in practice is challenging because it requires a long-term commitment from the pet owner to complete the protocol. Based on review of purchase records alone, over half of the dogs in this study did not complete the SK protocol as prescribed (Table 1). Because the preventive used in SK is intended to protect against future $D$. immitis infection as well as clear existing nematodes ${ }^{15}$, some of the dog in the present study also may have been reinfected due to poor or non-compliance; this reinfection and subsequent development of adult heartworms may have contributed to the presence of blocked antigen in some patients which was revealed only upon heat treatment of serum. Nonetheless, several dogs being managed with SK tested false negative on standard commercial antigen tests, indicating that many negative antigen test results from dogs on SK may not be accurate.

\section{Conclusions}

Managing $D$. immitis-infected dogs with monthly macrocyclic lactones and doxycycline can create false negative results on heartworm antigen tests, misleading veterinarians and pet owners about the efficacy of this approach. The data from the present study indicate that antigen detection as a primary outcome measure is not an accurate means of determining SK success, suggesting previously reported efficacy of SK in pet dogs in clinical practice should be reconsidered. Adulticide therapy with melarsomine dihydrochloride remains the treatment recommended by the Companion Animal Parasite Council, the European Scientific Counsel Companion Animal Parasites, and the American Heartworm Society for dogs infected with adult heartworms.

\section{Competing interests}

This study was supported by Novartis Animal Health, of which JD and LA are employees. 


\section{Authors' contributions}

JD conceived and helped design the study and participated in the drafting and revision of the manuscript. SL led the design and conduct of the study and participated in the acquisition, analysis and interpretation of the data and revision of the manuscript. JG and HM coordinated acquisition and testing of the majority of the sample included in the study. LA participated in field activities, identification and enrollment of cases as well as revision of the manuscript. All authors read and approved the final manuscript.

\section{Acknowledgments}

The authors are very grateful to the practicing veterinarians who participated in this study by providing samples and access to medical records for review.

\section{Author details}

${ }^{1}$ Novartis Animal Health, Greensboro, NC, USA. ${ }^{2}$ Department of Veterinary Pathobiology, Center for Veterinary Health Sciences, Oklahoma State University, Room 25074078 McElroy Hall, Stillwater, OK, USA. ${ }^{3}$ Swaim Serum Company, Oklahoma City, OK, USA.

Received: 4 December 2014 Accepted: 26 January 2015 Published online: 04 February 2015

\section{References}

1. Nelson CT, McCall J, Carithers D. Current canine guidelines for the diagnosis, prevention, and management of heartworm (Dirofilaria immitis) infection in dogs (revised July 2014). wuw.heartwormsociety.org, accessed December 1, 2014.

2. Companion Animal Parasite Council. CAPC General Guidelines, 2014. http://www.capcvet.org/capc-recommendations/capc-general-guidelines, accessed December 1, 2014

3. European Scientific Councel Companion Animal Parasites. Control of VectorBorne Diseases in Dogs and Cats. ESCCAP Guideline 05 Second Edition October 2012. http://www.esccap.org, accessed December 1, 2014.

4. Rawlings CA, Bowman DD, Howerth EW, Stansfield DG, Legg W, Luempert LG. Response of dogs treated with ivermectin or milbemycin starting at various intervals after Dirofilaria immitis infection. Vet Ther. 2001;2:193-207.

5. Bowman DD, Atkins CE. Heartworm biology, treatment, and control. VCNA: Small Anim Pract. 2009;39:1127-58.

6. Keith JC, Schaub RG, Rawlings C. Early arterial injury-induced myointimal proliferation in canine pulmonary arteries. Am J Vet Res. 1983;44:181-6.

7. Rawlings CA, McCall JW, Lewis RE. The response of the canine heart and lungs to Dirofilaria immitis. J Am Anim Hosp Assoc. 1980;14:17-32.

8. Schaub RG, Rawlings CA. Pulmonary vascular response during phases of canine heartworm disease: scanning electron microscopic study. Am J Vet Res. 1980;41:1082.

9. Sutton RH. Pathology and pathogenesis of dirofilariasis. In: Borehan A, editor. Dirofilariasis. Boca Raton, FL: CRC Press; 1988. p. 99-132.

10. Miller MW, Keister DM, Tanner PA, Meo NJ. Clinical efficacy of melarsomine dihydrochloride (RM340) and thiacetarsamide in dogs with moderate (Class 2) heartworm disease. Auburn, Alabama, USA: Proceedings of the Heartworm Symposium '95; 1995. p. 233-41.

11. McCall JW, Ryan WG, Roberts RE, Dzimianski MT. Heartworm adulticidal activity of prophylactic doses of ivermectin $(6 \mu \mathrm{g} / \mathrm{kg})$ plus pyrantel administered monthly to dogs. Batavia, IL, USA: Recent Advances in Heartworm Disease: Symposium'98, American Heartworm Society; 1998. p. 209-15.

12. McCall JW, Guerrero J, Roberts RE, Supakorndej N, Mansour AE, Dzimianski MT, et al. Further evidence of clinical prophylactic, retro-active (reach-back) and adulticidal activity of monthly administrations of ivermectin (Heartgard Plus ${ }^{\text {TM}}$ ) in dogs experimentally infected with heartworms. Batavia, IL, USA: Recent Advances in Heartworm Disease: Symposium'01, American Heartworm Society; 2001. p. 189-200.

13. Passeri B, Vismarra A, Cricri G, Bazzocchi C, Kramer L, Bacci C. The adulticide effect of a combination of doxycycline and ivermectin in Dirofilaria immitis-experimentally infected dogs is associated with reduction in local T regulatory cell populations. Vet Parasitol. 2014. in press.

14. Kosić LS, Lalosević V, Simin S, Kuruca L, Naglić A, Vasić I. Microfilaremia and antigenemia in dogs with natural heartworm infection treated with combination of doxycycline and ivermectin - prelimilary results. Budapest, Hungary: Fourth European Dirofilaria and Angiostrongylus Days (FEDAD); 2014. p. 62
15. Bowman DD, Mannella C. Macrocyclic lactones and Dirofilaria immitis microfilariae. Topic Compan Anim Med. 2011;26:160-72.

16. Bourguinat C, Keller K, Bhan A, Peregrine A, Geary T, Prichard R. Macrocyclic lactone resistance in Dirofilaria immitis. Vet Parasitol. 2011;181:388-92.

17. Geary TG, Bourgionat C, Blagburn BL, Keller K, Schenker R, Prichard RK. Genetic changes in Dirofilaria immitis populations possibly associated with exposure to macrocyclic lactones. Atlanta, GA, USA: American Association of Veterinary Parasitologists, 55th Annual Meeting; 2010. p. 62.

18. Prichard RK, Bourguinat C, Geary TG, Keller K, Blagburn B, Bowman DD, et al. Markers for macrocyclic lactone resistance in Dirofilaria immitis and utilization for management of heartworm disease. Chicago, IL, USA: American Association of Veterinary Parasitologists, 58th Annual Meeting; 2013. p. 63-4.

19. Velasquez L, Blagburn BL, Duncan-Decoq R, Johnson EM, Allen KE, Meinkoth J, Gruntmeir J, Little SE. Increased prevalence of Dirofilaria immitis antigen in canine samples after heat treatment. Vet Parasitol. 2014. in press.

20. Little SE, Raymond MR, Thomas JE, Gruntmeir J, Hostetler JA, Meinkoth JH, et al. Heat treatment prior to testing allows detection of antigen of Dirofilaria immitis in feline serum. Parasit Vectors. 2014;7:1.

21. Little SE, Munzing C, Heise SR, Allen KE, Starkey LA, Johnson EM, et al. Pre-treatment with heat facilitates detection of antigen of Dirofilaria immitis in canine samples. Vet Parasitol. 2014;203:250-2.

22. Zajac AM, Conboy GA. Detection of parasites in the blood. Veterinary Clinical Parasitology. 8th ed. Ames, IA, USA: Wiley-Blackwell; 2012. p. 185-211.

23. Rishniw M, Barr SC, Simpson KW, Frongillo MF, Franz M, Alpizar JLD. Discrimination between six species of canine microfilariae by a single polymerase chain reaction. Vet Parasitol. 2006;135:303-14.

24. Venco L, McCall JW, Guerrero J, Genchi C. Efficacy of long-term monthly administration of ivermectin on the progress of naturally acquired heartworm infections in dogs. Vet Parasitol. 2004;124:259-68.

25. Weil GJ, Malane MS, Powers KG. Detection of circulating parasite antigens in canine dirofilariasis by counterimmunoelectrophoresis. Am J Trop Med Hyg. 1984:33:425-30.

26. Tonelli QJ. Factors affecting the accuracy of enzyme immunoassays for Dirofilaria immitis adult antigen. In: Seward RL, editor. Proceedings of the American Heartworm Symposium. Batavia, IL, USA: American Heartworm Society; 1989. p. 161-5.

27. Blagburn B, Bowles J, Loechel R, Carmichael J, Schenker R, Roycroft L. Evidence of genetic selection following treatment of a heartworm-infected, microfilaremic dog with increasing doses of ivermectin. Chicago, IL, USA: American Association of Veterinary Parasitologists, 58th Annual Meeting; 2013. p. 64.

28. McCall JW, Genchi C, Kramer L, Guerrero J, Dzimianski MT, Supakorndej P, et al. Heartworm and Wolbachia: therapeutic implications. Vet Parasitol. 2008;158:204-14.

29. McCall JW, Kramer L, Genchi C, Guerrero J, Dzimianski MT, Mansour A, et al. Effects of doxycycline on heartworm embryogenesis, transmission, circulating microfilaria, and adult worms in microfilaremic dogs. Vet Parasitol. 2014;206:5-13.

\section{Submit your next manuscript to BioMed Central and take full advantage of:}

- Convenient online submission

- Thorough peer review

- No space constraints or color figure charges

- Immediate publication on acceptance

- Inclusion in PubMed, CAS, Scopus and Google Scholar

- Research which is freely available for redistribution 